\title{
Integrated approaches to the optimisation of regional and local food-energy-water systems
}

\author{
Anton Johannes Veldhuis, Aidong Yang \\ Department of Engineering Science, University of Oxford, Parks Road, Oxford OX1 3PJ, UK
}

Corresponding author: Yang, Aidong (aidong.yang@eng.ox.ac.uk)

\begin{abstract}
Optimisation of regional and local food-energy-water systems that takes into account the connections between the three sectors is classified into "integration based on footprint" and "integration based on co-decision". Footprint-based approaches focus on the decision on one main system while using static footprint data to account for the links with other systems. In contrast, codecision based approaches aim to simultaneously optimise multiple, interconnected systems. Choosing between these two approaches needs to consider the nature of the interactions between different systems as well as data availability and the feasibility of implementing the optimisation results. Future research in this area is expected to broaden decision targets and to improve the handing of the multiple scales involved in integrated food-energy-water decision making.
\end{abstract}

\section{Introduction}

Food, energy and water are among the most essential requirements for the thriving of every society. Although conventionally they are supplied by different economic sectors, the challenges facing their secure supply are highly interconnected, due to the significant energy and water consumption in food production, the mutual footprint between energy and water production, and the intertwined connections of the three sectors with land and the broader ecosystems. The interconnectedness between the three sectors has been coined the term "food-energy-water (FEW) nexus", as a concept helping to raise awareness of the inseparable challenges and to frame the needs for an integrative approach [1, 2].

In recent years, tools to support systematic decision making on FEW systems have emerged, aiming to address the nexus challenges at global, regional and local scales. A number of tools have been developed for the modelling and assessment of the performance of FEW systems [3-5]. Another type of tools, with a nature of optimisation, attempts to directly suggest optimal designs, plans or operational strategies. Most of the existing optimisation work on FEW nexus has been carried out for the regional scale, with a few studies focusing on local systems which can be of special interest for exploring potentials of distributed or decentralised economic schemes [6, 7].

The purpose of this paper is to provide a concise review of the integrated approaches for optimising regional and local FEW systems. In particular, a distinction is made between integration based on footprint (IbF) and integration based on co-decision (IbC). The approaches are different in the level of sophistication in capturing the interactions between the FEW systems. This brief review will summarise and contrast these two approaches, and discuss common issues facing FEW optimisation, such as decision targets, scales and resolutions, with an aim to identify key avenues for future research in this area.

\section{Integration based on footprint (IbF)}

Footprints are quantities representing the implications of operating one system to the others, and have been widely used in sustainability assessment. In particular, water-, energy- and land-footprints, among other footprints reviewed by Čuček et al. [8], specify the level of consumption of these resources for producing a unit amount of output by a relevant system and can be used to quantify the inter-system connections in the FEW nexus. As illustrated in Figure 1, The IbF approach focuses decisions on a main system while taking into account the impacts and limitations pertaining to other 
systems (through either inter-system connections or interactions with common resources shared by different systems) by means of footprints. IbF has typically been carried out by mathematical programming, in which footprints are incorporated through objective functions and constraints.

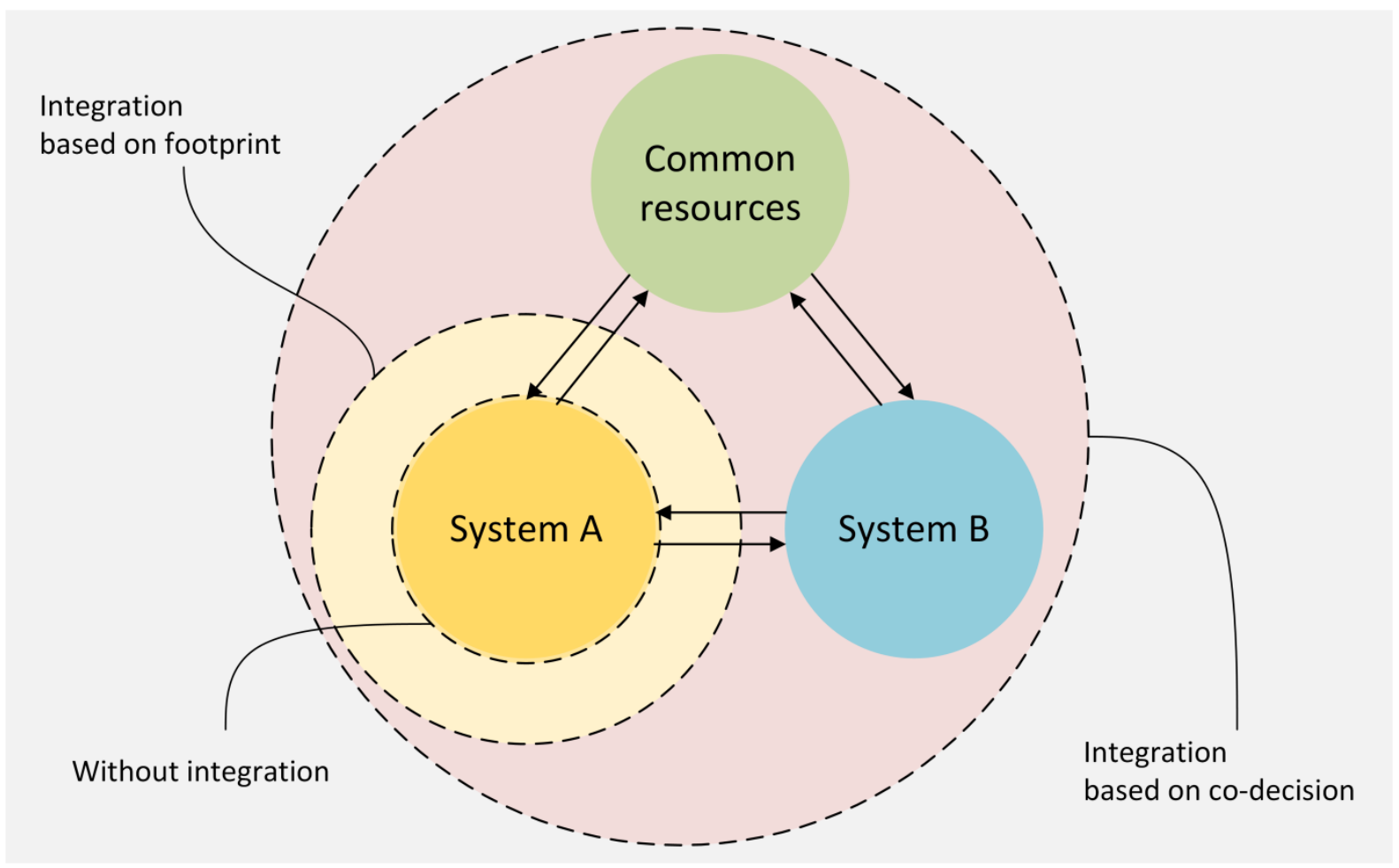

Figure 1: Different optimisation approaches: optimisation without integration, integration based on footprint, and integration based on co-decision. Boundaries of these approaches are marked by dashed circles.

This approach has been widely applied to the optimisation of energy systems, which takes into account water consumption and other factors such as land use and ecosystem impacts. In particular, a number of applications are found in the area of regional supply chain optimisation for bioenergy [9]. As a representative example, Čuček et al. [10] optimised technical decisions for producing fuels, electricity, heat and other material products from regional biomass resources. Multiple economic, environmental and social objectives were considered, including economic cost, water consumption and water pollution, land competition between food and energy crops, and the lost opportunities for food and feed supply due to the conversion of multi-purpose crops to energy products. In other work, water footprints have been incorporated either through objective functions (e.g. maximisation of economic or energy output per unit water consumption, [11]) or constraints (e.g. water supply limit, [12]; water stress, defined as the ratio of water withdrawal rate to water availability, [13]). Various constraints have also been used to introduce land footprints to account for the potential conflict between food and energy [14, 15]. In addition to bioenergy systems optimisation, IbF has also been used in designing energy systems with combined use of renewable and fossil resources, considering both water and land footprints [16], and in designing systems based on fossil fuels [17].

A number of studies using IbF exist with a focus on agricultural systems, which determine the optimal mix of crops for a specific region and the corresponding water (and land) allocation, typically for economic objectives (e.g. $[18,19])$. Such an optimisation problem requires water consumption data for each crop, as well as the availability of different water resources and limits to be imposed on 
agricultural water use. The decisions may be made considering not only water consumptions, but also the further ecosystem and resource impacts of water depletion, as in the work of Galán-Martín et al. [20], which also adopted the full concept of water footprint [21].

\section{Integration based on co-decision (IbC)}

Different from IbF, where considerations on other system(s) are incorporated into the optimisation of a main system through footprint data, IbC aims to co-optimise multiple systems, with comparable considerations of these systems in objectives and decision variables, and incorporating similar levels of detail for their structural and/or behavioural characteristics (cf. Figure 1).

A number of applications of IbC have been reported for the optimisation of energy-water systems [22]. As an representative example, the work by Dubreuil et al. [23] explored joint decision making in the multi-national water-scarce Middle East region. An existing energy optimisation model (TIAMTR) was combined with a newly developed model for water systems. Each model contained details of the sources, processing/conversion technologies and distribution. The overall optimisation problem was to minimise the total discounted cost for energy (including energy consumption for water production) by choosing the best primary sources, technologies, and allocation of water and energy to various socio-economic sectors, subject to the constraints on the availability of primary sources. The work of Bhatt et al. [24], addressing a much smaller geographical scope at the city level, extended the established MARKAL energy model to include a water-system counterpart. The combined model was able to minimise the total economic cost by choosing optimal technical options in both systems. The model was used to support urban planners to study a number of scenarios with respect to energy and water savings and carbon emission reduction. Further examples include the work by Parkinson et al. [25] with a purposely-built integrated model for national energy and water systems in Saudi-Arabia, and work for regions with competing uses of water between irrigation and hydropower, such as [23].

To-date, studies on co-decision of all the three systems (food, energy and water) are still rare, but emerging. Zhang and Vesselinov [26] presented an optimisation model with an aim to cover simultaneously food, energy and water production. A greater level of detail was given to the energy (coal and natural gas based power generation) and water (supply of groundwater and surface water; generation of recycled water) systems, while the food system was rather simplified with no details given to product types or the supply chain. Nevertheless, the optimisation aimed to simultaneously satisfy the social demands of all three commodities. In a work focusing on the integrated design of local FEW systems [27], a mathematical programming based approach has been developed to minimise the total resource cost (in cumulative exergy consumption, [28]) while observing constraints of local resource (land, renewable energy, biomass, and water) availability. A case study on a UK eco-town included multiple food types and their production/processing chains, options for heat and power generation based on local renewables, and water supply from a range of sources. The overall optimisation model does not only represent the inter-system energy and water flows, but also explores the opportunities for symbiotic resource (e.g. heat, residual streams) reuse between and within FEW systems.

It is worth noting that, the last work and the others reviewed earlier are mostly based on mathematical programming, which relies on formulating the design problem into one of mathematical optimisation and subsequently solving the problem with an appropriate optimisation algorithm. In a more recent extension, a so-called insight-based approach has been developed which solves the problem of designing a local FEW system primarily through a multi-stage iterative procedure that is based on design rules and principles [29]. As in similar methods already applied for designing chemical process systems (e.g. [30]), this approach has the potential to offer a more flexible and transparent decision process which better integrates the input from and provides further insights to the decision makers. It avoids the formulation of sophisticated mathematical models, but still allows the use of relatively 
straightforward mathematical optimisation techniques (e.g. linear programming) to be used in solving well-defined sub-problems, as demonstrated in [29].

\section{Choosing between IbF and IbC}

Compared to IbF, IbC can be significantly more sophisticated, by incorporating the objectives, decision variables, causal relationships and other details from multiple systems, as opposed to a single main system. IbC becomes necessary when important feedback mechanisms exist (e.g. energy generation decisions affect water decisions which in turn affect cost and/or availability of water for energy, which needs to be considered in energy generation decisions hence forming a feedback loop), or when resource sharing between multiple systems is significantly affected by decisions from all systems (e.g. land distribution between food and energy, where the land use intensity of each activity is not a constant but rather depends on the decisions in the sector where this activity takes place). As footprint accounting is based on the constant footprint parameters, IbF is unsuitable to handle the above cases.

On the other hand, the necessity of adopting IbC needs to be counter-balanced with the feasibility associated with its complexity, particularly in terms of data availability (as multiple systems are now to be modelled in detail) and practical adoptability (as multiple stakeholders are required to synchronise their actions in order to sensibly implement the optimisation results). When there is a lack of mechanisms to effectively draw multiple sectors into a framework of joint data collection and decision implementation, IbF led by a single sector would become the more practical option.

To further appreciate the challenges in data collection, one needs to realise that a broad range of data may be required to support optimisation and other types of study on the FEW nexus, including climate, resource availability, resource consumption or efficiency, environmental impact and economics. Furthermore, these data may be required at a wide spectrum of resolution, from global to national and local and from the whole economy to sectors, technologies and specific processes. At present, these data are rather scattered in different sources, such as the databases maintained by international organisations (e.g. the AQUASTAT database of FAO on water consumption and availability, www.fao.org/nr/aquastat), national agencies (e.g. databases of US Energy Information Agency on energy generation, consumption and efficiency, www.eia.gov), collaborative networks (e.g. the data sets on water footprint by the Water Footprint Network, www.waterfootprint.org) and life-cycle analysis (LCA) software. On a more ad-hoc basis, data are also reported by commissioned studies or research papers on specific sectors or products (e.g. the energy efficiency report by Carbon Trust for industrial bakeries, www.carbontrust.com; assessment of packaged tomato puree, [31]). To support integrated studies on the FEW nexus, tools need to be developed to enable researchers and practitioners to efficiently navigate between, connect and access existing sources and to generate and integrate new data. As in dealing with any complex system, availability of sufficient, reliable and consistent data constitutes a key challenge and hence a research priority in this area.

\section{Defining decision targets: security, sustainability and resilience}

In both $\mathrm{IbF}$ and IbC, multiple decision targets are often considered through objective functions or constraints. Examined against a security-sustainability-resilience framework (Figure 2), virtually all optimisation work reviewed earlier has aimed to meet demands through sufficient production/generation, and many have also considered proper distribution of products, corresponding respectively to availability and physical accessibility of products and services, which are two important aspects of security [32]. Economic accessibility, another aspect of security, has been addressed to some extent through cost minimisation. While an optimisation problem can be formulated such that these security targets are directly pursued for a defined time span, long-term security has to be enabled through sustainability. Sustainability targets have so far been incorporated in a rather ad-hoc manner and mainly limited to environmental impacts, through accounting for 
greenhouse gas emission (e.g. carbon footprint) and local impacts such as those associated with water depletion (e.g. [20]) and with changes in land use (e.g. [15]). Another category of targets, which has been least addressed, covers the capability of the FEW systems to achieve security under future climatic, economic, social and political uncertainties, which is broadly referred to as resilience [33]. Various resilience attributes have been defined for FEW sectors (e.g. [33-36]); future FEW optimisation could explore the possibility of incorporating such attributes into objective functions or constraints to find decisions that can explicitly strengthen resilience. This approach can complement optimisation techniques for coping with uncertainties, such as stochastic programming and robust optimisation [37]; see also [38] for the potential applications of these techniques in FEW nexus.

\section{FEW systems' outcome:}

(availability \& accessibility of products and services)

\section{Security}

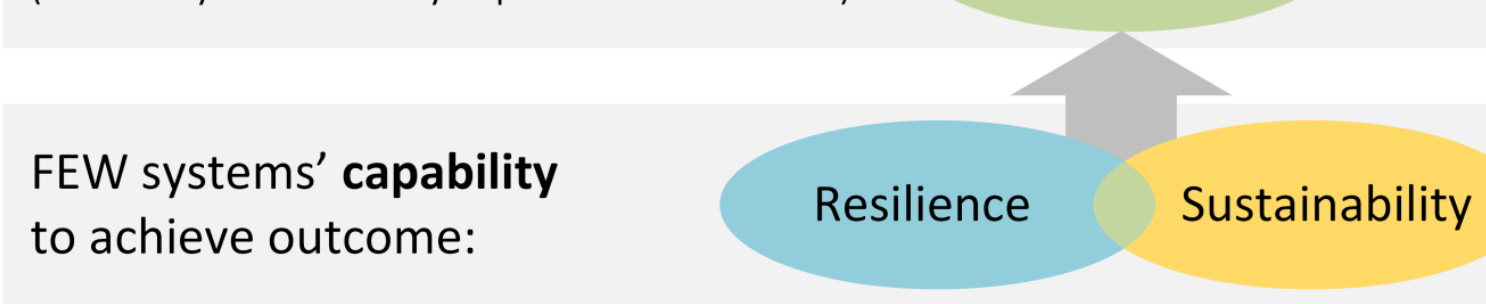

Figure 2: Targets for FEW optimisation: security, resilience and sustainability.

\section{Dealing with scale and resolution}

\section{Scales and resolutions for FEW nexus decision}

FEW optimisation has so far been carried out at multi-national, national and local scales. At larger spatial scales (e.g. multi-national, national, or regional in a large country), the problem formulation or solution usually refers to types of technical options or products and their (aggregated) capacities or volumes, instead of specific instances of facilities or flows as in the case of a local system optimisation. On the other hand, optimisation at a large scale may attempt to enhance the inclusion of geographically-dependent details such as elevation, soil productivity and demands, through choosing a proper spatial resolution corresponding to a lower scale, e.g. watershed [20] or province [25]. Data adopted at such a resolution could have been obtained originally through aggregating information available at an even finer resolution [20], although the trade-off between model quality and computational complexity may still favour the direct use of the coarser resolution in the optimisation model.

Along the temporal dimension, optimisation involving capital investment typically considers a time scale of multiple decades, within which a monthly or seasonal resolution is often adopted to account for important variations in demand and supply. One exception is the work of Santhosh et al. [39] on real-time dispatch of power and water, which adopts an hourly resolution within the time span of a day.

Dealing with the multiple spatial, organisational and temporal scales has been recognised as a major challenge for FEW nexus [5], two aspects of which are illustrated in Figure 3 and briefly discussed below. 


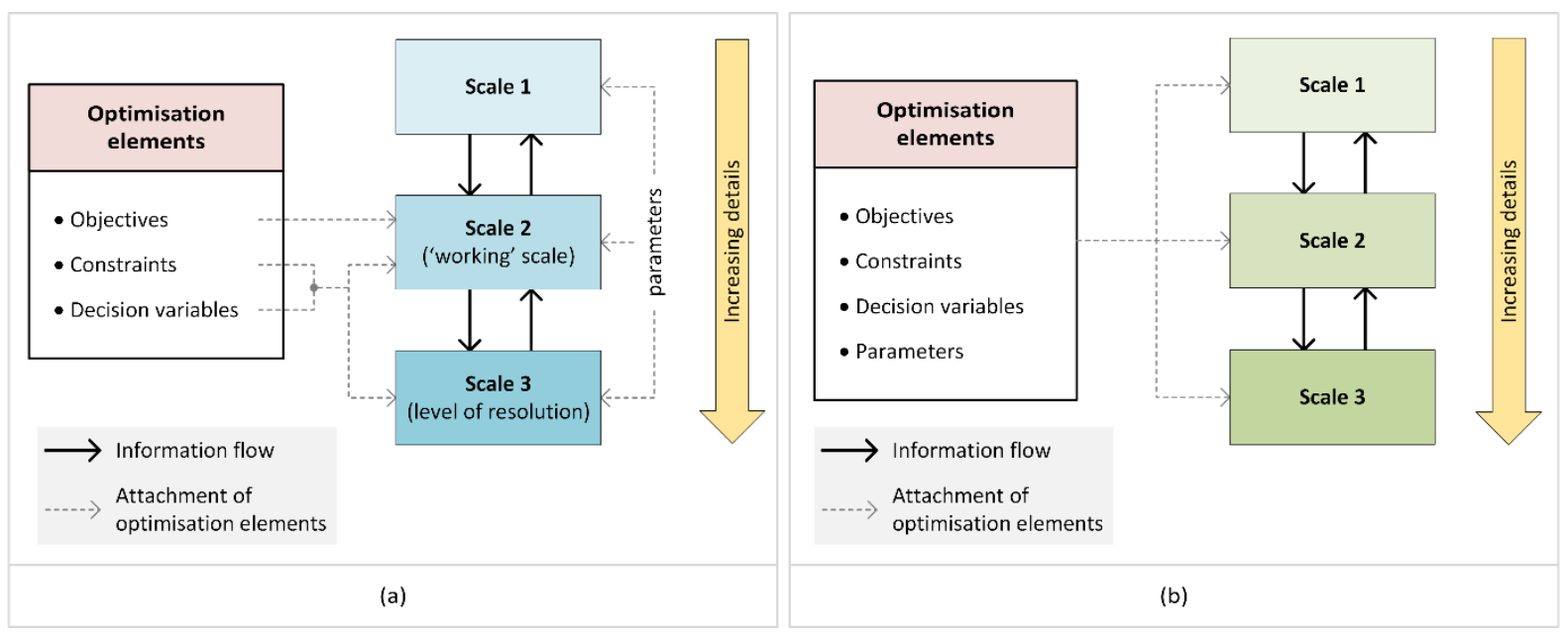

Figure 3: Dealing with scales and resolutions in FEW optimisation. (a) Decision making at a suitable "working" scale, where a lower scale may be taken to define the resolution for modelling or information collection; (b) Decision making across multiple scales.

\section{Choosing a working scale for decision making}

Some decision tasks require choosing a suitable "working" scale at which the problem should be defined and solved, particularly in terms of the introduction and quantification of decision objective(s). Given the nature of a decision problem, the "working" scale should be chosen to allow all the influencing factors, mechanisms and important effects to be encompassed, while taking into account practical constraints such as data availability and adoptability of resulting decisions, similar to the constraints for the application of IbC discussed earlier. For example, the attachment of multiple countries to a regional water resource dictates that the optimal planning be considered at an international (instead of national) level; yet a corresponding mechanism at this level needs to exist to allow the collection of multi-national data and to eventually implement the decisions. It should be noted that, working at a suitable scale does not imply the neglect of other scales, since information and impacts do cross scale boundaries. Multi-scale information aggregation and disaggregation, suggested by Hanes and Bakshi [40], may prove useful in informing the decision at one scale (e.g. factory) with information available at higher scales (e.g. supply chain), which is particularly relevant to IbF, and in assessing the impact of a decision at a higher level (e.g. a national policy) on an entity at a lower level (e.g. a factory).

\section{Decision making with multiple scales}

Another aspect of the multi-scale challenge, beyond decision making at a (single) working scale, stems from the need to recognise and ideally harmonise political, economic, social powers (corresponding to decision variables) and interests (corresponding to objectives and constraints) associated with different geographical/organisational scales within the FEW nexus. A relevant approach to this need is multi-level (or bi-level for a two-level system) optimisation [41], which recognises existing decision hierarchies and allows decisions to be made at different levels, each with distinguished objective(s), while considering cross-level obligations (i.e. conditions resulting from a higher-level decision, to be honoured at a lower-level) and consequences (i.e. factors to be considered by a higher-level decision which are due to the decisions at a lower-level). This approach has recently been applied to the optimisation of the energy-water nexus [17]. The bi-level optimisation framework assumes the presence of a leader and a follower, which may be applicable to the FEW nexus under certain circumstances, such as those involving the interactions between national policy makers and local companies or farmers. 
However, there are other circumstances where a higher-level decision is pursued not as part of the operation within a pre-defined vertical power structure, but rather to re-conciliate overlapping or conflicting interests of multiple parties within the same scale (or level of power), as in the case of the Water and Energy Uses Round Table formed by countries of the Syr Darya Basin [23]. Hierarchical optimisation, as applied by Singh and Lou [42] to industrial ecosystems, is capable of optimising multiple subsystems with conflicting objectives which, however, are still expected to readily accept the "co-ordination" from the higher-level for the sake of a global optimisation. To deal with cases where the decision makers are not "co-operative", game theoretic approaches have previously been introduced to supply chain optimisation [43], which may prove useful also for FEW decisions. Furthermore, bottom-up approaches suitable for assessing the impact of independent decisions, such as agent-based modelling as previously proposed for studying regional energy networks [44], could prove complementary to conventional, top-down optimisation approaches.

\section{Conclusion}

Existing work on the optimisation of regional and local FEW systems has been based on either footprint or co-decision. While the footprint-based approach is capable of taking into account of static impact of one system on the others, the co-decision approach is required to sufficiently account for the feedback mechanisms and the sharing of common resources within the FEW nexus. The application of the latter approach is currently rather limited. On the decision targets, there is scope for a greater and more systematic coverage of all the important security, sustainability and resilience dimensions. Furthermore, much of the complexity in FEW decisions lies in (1) the selection of suitable "working" scales for making decisions and suitable resolutions for modelling and information collection, and (2) the connection of objectives, constraints, variables and mechanisms across multiple scales. While scale-related challenges have so far been explicitly addressed in FEW optimisation only to a very limited extent, approaches and techniques such as multiscale modelling, multi-level/hierarchical optimisation and combination of top-town and bottom-up approaches, which have been applied to other related areas, can be taken as the basis for further development and application in FEW nexus. In dealing with the above challenges, the availability of sufficient reliable and consistent data is often a prerequisite, which represents a priority for the work in this area.

As a final note, most of the optimisation work reviewed in this paper was for supporting only technical decisions. In reality, there are certainly other types of decisions for the FEW systems, ranging from policies to business models, which may be jointly considered along with technical decisions in the future.

\section{Acknowledgements}

This work has been supported by the Leverhulme Trust (RPG-2012-663) and UK's Engineering and Physical Sciences Research Council (EP/M017753/1).

\section{References}

Papers of particular interest have been highlighted as:

- of special interest

$\bullet \bullet$ of outstanding interest

1. Hoff, H., Understanding the nexus. Background paper for the Bonn conference on the waterfood-energy nexus. 2011, SEl Stockholm.

2. Flammini, A., et al., Walking the nexus talk: assessing the water-energy-food nexus in the context of the sustainable energy for all initiative. 2014: FAO.

3. Daher, B.T. and R.H. Mohtar, Water-energy-food (WEF) Nexus Tool 2.0: guiding integrative resource planning and decision-making. Water International, 2015. 40(5-6): p. 748-771. 
4. Howells, M., et al., Integrated analysis of climate change, land-use, energy and water strategies. Nature Climate Change, 2013. 3(7): p. 621-626.

5. Keairns, D., R. Darton, and A. Irabien, The energy-water-food nexus. Annual review of chemical and biomolecular engineering, 2016. 7: p. 239-262.

6. Johansson, A., P. Kisch, and M. Mirata, Distributed economies-a new engine for innovation. Journal of Cleaner Production, 2005. 13(10): p. 971-979.

7. Martinez-Hernandez, E., et al., A Framework for Modeling Local Production Systems with Techno-Ecological Interactions. Journal of Industrial Ecology, 2016.

8*. Čuček, L., J.J. Klemeš, and Z. Kravanja, A review of footprint analysis tools for monitoring impacts on sustainability. Journal of Cleaner Production, 2012. 34: p. 9-20.

This paper presents a comprehensive review of footprints defined for quantifying the sustainability of production systems.

9. Yue, D., F. You, and S.W. Snyder, Biomass-to-bioenergy and biofuel supply chain optimization: overview, key issues and challenges. Computers \& Chemical Engineering, 2014. 66: p. 36-56.

10. Čuček, L., et al., Total footprints-based multi-criteria optimisation of regional biomass energy supply chains. Energy, 2012. 44(1): p. 135-145.

This paper offers a detailed example of integrated optimisation of bioenergy systems considering water and land footprints.

11. Garcia, D.J. and F. You, Life cycle network modeling framework and solution algorithms for systems analysis and optimization of the water-energy nexus. Processes, 2015. 3(3): p. 514539.

12. You, F., et al., Optimal design of sustainable cellulosic biofuel supply chains: multiobjective optimization coupled with life cycle assessment and input-output analysis. AIChE Journal, 2012. 58(4): p. 1157-1180.

13. Bernardi, A., S. Giarola, and F. Bezzo, Optimizing the economics and the carbon and water footprints of bioethanol supply chains. Biofuels, Bioproducts and Biorefining, 2012. 6(6): p. 656-672.

14. Čuček, L., et al., Multi-period synthesis of optimally integrated biomass and bioenergy supply network. Computers \& Chemical Engineering, 2014. 66: p. 57-70.

15. Guo, M., et al., Implementing land-use and ecosystem service effects into an integrated bioenergy value chain optimisation framework. Computers \& Chemical Engineering, 2016. 91: p. 392-406.

16. Kursun, B., et al., Life cycle and emergy based design of energy systems in developing countries: Centralized and localized options. Ecological Modelling, 2015. 305: p. 40-53.

17. Zhang, X. and V.V. Vesselinov, Energy-water nexus: Balancing the tradeoffs between twolevel decision makers. Applied Energy, 2016. 183: p. 77-87.

18. Xevi, E. and S. Khan, A multi-objective optimisation approach to water management. Journal of environmental management, 2005. 77(4): p. 269-277.

19. Singh, A., Optimal allocation of resources for the maximization of net agricultural return. Journal of Irrigation and Drainage Engineering, 2012. 138(9): p. 830-836.

20. Galán-Martín, Á., et al., Multi-objective optimization of rainfed and irrigated agricultural areas considering production and environmental criteria: a case study of wheat production in Spain. Journal of Cleaner Production, 2017. 140: p. 816-830.

This paper represents a modelling framework for optimising water and land allocation for agricultural production with considerations in ecosystems and resource impact of water depletion and using the full concept of water footprint.

21. Aldaya, M.M., et al., The water footprint assessment manual: Setting the global standard. 2012: Routledge. 
22. Khan, Z., P. Linares, and J. García-González, Integrating water and energy models for policy driven applications. A review of contemporary work and recommendations for future developments. Renewable and Sustainable Energy Reviews, 2017. 67: p. 1123-1138.

This paper reviews approaches and application of joint decision making on energy and water systems.

23. Dubreuil, A., et al., Water modeling in an energy optimization framework-The water-scarce middle east context. Applied energy, 2013. 101: p. 268-279.

24. Bhatt, V., et al., NEW YORK CITY ENERGY-WATER INTEGRATED PLANNING: A PILOT STUDY. 2008, Brookhaven National Laboratory.

25. Parkinson, S.C., et al., Impacts of Groundwater Constraints on Saudi Arabia's Low-Carbon Electricity Supply Strategy. Environmental science \& technology, 2016. 50(4): p. 1653-1662.

26. Zhang, X. and V.V. Vesselinov, Integrated modeling approach for optimal management of water, energy and food security nexus. Advances in Water Resources, 2017. 101: p. 1-10.

This is one of the first work on the co-decision of all three (food, energy and water) systems.

$27^{\circ}$. Leung Pah Hang, M.Y., et al., Designing integrated local production systems: $A$ study on the food-energy-water nexus. Journal of Cleaner Production, 2016. 135: p. 1065-1084.

This is the first work on the integrated design of local food-energy-water systems that accounts for the detailed exchange of flows and symbiotic resource reuses.

28. Sciubba, E. and G. Wall, A brief commented history of exergy from the beginnings to 2004. International Journal of Thermodynamics, 2007. 10(1): p. 1-26.

29. Leung Pah Hang, M.Y., et al., Insight-Based Approach for the Design of Integrated Local FoodEnergy-Water Systems. Environmental Science \& Technology, 2017. 51(15): p. 8643-8653.

30. Foo, D.C., Process integration for resource conservation. 2012: CRC Press.

31. Manfredi, M. and G. Vignali, Life cycle assessment of a packaged tomato puree: a comparison of environmental impacts produced by different life cycle phases. Journal of Cleaner Production, 2014. 73: p. 275-284.

32. Willis, H.H., et al., Developing the Pardee RAND Food-Energy-Water Security Index. 2016.

33. Toth, A., S. Rendall, and F. Reitsma, Resilient food systems: a qualitative tool for measuring food resilience. Urban Ecosystems, 2016. 19(1): p. 19-43.

This paper contains useful discussions on the distinction between security and resilience, in the context of food systems.

34. Gracceva, F. and P. Zeniewski, A systemic approach to assessing energy security in a lowcarbon EU energy system. Applied Energy, 2014. 123: p. 335-348.

35. Asefa, T., et al., Performance evaluation of a water resources system under varying climatic conditions: Reliability, Resilience, Vulnerability and beyond. Journal of Hydrology, 2014. 508: p. 53-65.

36. Chen, C.N., I.; Hellmann, J.; Coffee, J.; Murillo, M.; Chawla, N. , University of Notre Dame Global Adaptation Index - Country Index Technical Report 2015, University of Notre Dame

37. Mulvey, J.M., R.J. Vanderbei, and S.A. Zenios, Robust optimization of large-scale systems. Operations research, 1995. 43(2): p. 264-281.

38*. Garcia, D.J. and F. You, The water-energy-food nexus and process systems engineering: $A$ new focus. Computers \& Chemical Engineering, 2016. 91: p. 49-67.

This paper offers a comprehensive review of relevant approaches from process systems engineering to the studies of the food-energy-water nexus.

39. Santhosh, A., A.M. Farid, and K. Youcef-Toumi, Real-time economic dispatch for the supply side of the energy-water nexus. Applied Energy, 2014. 122: p. 42-52.

40. Hanes, R.J. and B.R. Bakshi, Process to planet: A multiscale modeling framework toward sustainable engineering. AIChE Journal, 2015. 61(10): p. 3332-3352.

41. Vicente, L.N. and P.H. Calamai, Bilevel and multilevel programming: A bibliography review. Journal of Global optimization, 1994. 5(3): p. 291-306. 
42. Singh, A. and H.H. Lou, Hierarchical pareto optimization for the sustainable development of industrial ecosystems. Industrial \& engineering chemistry research, 2006. 45(9): p. 32653279.

43. Yue, D. and F. You, Game-theoretic modeling and optimization of multi-echelon supply chain design and operation under Stackelberg game and market equilibrium. Computers \& Chemical Engineering, 2014. 71: p. 347-361.

44. Beck, J., et al., A complex systems approach to planning, optimization and decision making for energy networks. Energy policy, 2008. 36(8): p. 2795-2805. 